\title{
Exploiting Instruction Level Parallelism in Geometry Processing for Three Dimensional Graphics Applications
}

\author{
Chia-Lin Yang, ${ }^{1}$ Barton Sano, ${ }^{2}$ and Alvin R. Lebeck ${ }^{1}$ \\ ${ }^{1}$ Department of Computer Science \\ Duke University \\ Durham, North Carolina 27708 USA \\ \{yangc,alvy\}@cs.duke.edu \\ ${ }^{2}$ Western Research Lab \\ Compaq Corporation \\ sano@pa.dec.com
}

\begin{abstract}
Three dimensional (3D) graphics applications have become very important workloads running on today's computer systems. A cost-effective graphics solution is to perform geometry processing of $3 D$ graphics on the host $C P U$ and have specialized hardware handle the rendering task. In this paper, we analyze microarchitecture and SIMD instruction set enhancements to a RISC superscalar processor for exploiting instruction level parallelism $(I L P)$ in geometry processing for $3 D$ computer graphics. Our results show that $3 D$ geometry processing has inherent parallelism. When ignoring cycle time effects, an 8issue processor can achieve up to $60 \%$ performance improvement over a 4-issue. However, certain application attributes can hinder the exploitation of ILP on a superscalar processor. Adding SIMD operations improves performance from $8 \%$ to $28 \%$ on a 4-issue processor that can issue at most 2 floating-point operations. If processor cycle time scales with the number of ports to the register file, doubling only the floating-point issue width of a 4issue processor with SIMD instructions gives the best performance among the architecture configurations that we examine (the most aggressive configuration is an 8issue processor with SIMD instructions).
\end{abstract}

\section{Introduction}

The increasing number of multi-media applications produces a commensurate increase in demand for costeffective multi-media processing [10]. Traditionally, media processing was implemented in expensive custom

This work supported in part by NSF CAREER Award MIP-97-02547, ARPA Grant DABT63-98-1-0001, NSF Grants CDA-97-2637 and DA95-12356, Duke University, and an equipment donation through Intel corporation's Technology for Education 2000 Program. The views and conclusions contained herein are those of the authors and should not be interpreted as necessarily representing the official policies or endorsements, either expressed or implied, of the U.S. Government hardware specialized for specific applications (e.g., speech, video, and graphics). Advances in conventional microprocessor design now permit offloading some functionality to a general-purpose processor, possibly sacrificing performance in return for reduced cost. The key is to minimize this performance degradation, potentially by adding architectural support for media processing.

Many current microprocessors have Single Instruction Multiple Data (SIMD) type instructions to accelerate audio, video and 2D image processing, such as Intel MMX [15], Sun UltraSPARC VIS [9] and HP PA-RISC [8]. This type of SIMD instruction operates only on integer data. Today, several processor vendors, such MIPS Technology Inc. [14], Cyrix, IDT, AMD [2], Intel [6], and Motorola [18] are in various stages of incorporating floating-point SIMD instructions to speedup geometry processing for three dimensional (3D) graphics.

Typically, 3D graphics processing is a 3-stage pipeline [5]: 1) database traversal, 2) geometry computation, and 3) rasterization. Display models representing graphics scenes are generally stored in a database that must be traversed (stage 1) to extract the appropriate information for display, such as the drawing primitive (e.g., line or triangle), lighting models, etc. The information is then passed to the geometry subsystem (stage 2), which is responsible for transforming 3D coordinates to 2D coordinates. Finally, the rasterization stage (stage 3) converts transformed primitives into pixel values and stores them in the frame buffer for display.

In high-end graphics systems [16][17], the host CPU is only responsible for database traversal, and custom hardware is used for geometry processing and rasterization. The cost of building these high-end systems is generally too high for the mass market. To reduce cost, the host CPU could execute some, or all, of the graphics pipeline. This paper focuses specifically on host CPU execution of geometry computation using a single dynamically scheduled superscalar microprocessor. In particular, we examine the effects of microarchitectural changes and the bene- 


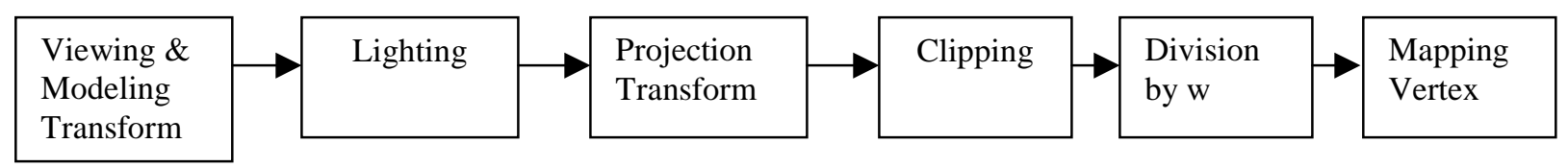

Figure 1: 3D geometry pipeline.

fits of recently proposed instruction set enhancements for geometry computation.

Geometry computation is floating-point intensive. Vertex coordinates, color and transformation matrices are stored in single-precision floating-point format. Geometry processing is an inherently parallel task, since each object vertex can be processed independently. Dynamically scheduled processors can exploit this parallelism by looking ahead in the instruction stream to identify and execute the operations associated with different vertices. Recall that vertex computations require only 32-bit floating-point values. Since most modern microprocessors have 64-bit floating-point registers, geometry calculations using 32-bit operands are utilizing only half the floating point datapath (registers, functional units, and busses). Another way to exploit this parallelism is using SIMD type instructions to perform operations on multiple vertices in one instruction-called paired-single instructions. Paired-single instructions fully utilize the 64-bit datapath by performing two independent 32-bit operations, each using half the datapath.

As mentioned above, most processor vendors are incorporating paired-single instructions. AMD's 3DNow! Technology [2] is currently available. However, we are unaware of any published quantitative evaluation of their performance using full applications. In this paper, we simulate Viewperf [19], an industry standard benchmark suite, on an out-of-order superscalar processor both with and without paired-single instructions. We modified the geometry computation routines in MESA [12] (a public domain implementation of OpenGL [24]) to utilize paired-single instructions. We first analyze the effects of increasing the resources available in a conventional processor for exploiting ILP. This is followed by a comparison to paired-single execution, both with and without clock cycle time effects.

The contributions of this paper are as follows:

(1) Although geometry processing presents substantial parallelism, we discover that certain aspects of application implementations can significantly impact the available instruction level parallelism (ILP) that can be exploited by a superscalar processor. In the best case, an 8-way issue processor can achieve $60 \%$ performance improvement over a 4 way with a 64-entry dispatch queue and 128 registers, but for certain benchmarks, the performance only increases by $20 \%$. Furthermore, if the CPU cycle time scales with the number of ports to the register file, the performance improvement is less than $5 \%$ for all the benchmarks.

(2) We analyze the effect of adding paired-single instructions on a set of industry standard 3D graphics benchmarks instead of small kernels. We found that the performance improvement from pairing up single-precision floating-point operations ranges from $8 \%$ to $28 \%$ on a 4 -way issue processor that can issue at most 2 floating-point operations per cycle.

(3) We quantify the benefits of paired-single instructions over increasing only the floating-point issue width in superscalar processors. Our results indicate that adding paired-single instructions to 4issue processor performs within $7 \%$ of doubling the floating-point issue width. For certain benchmarks, the former even outperforms the latter. The performance advantage of paired-single instructions increases when considering the clock cycle time effect.

The remainder of this paper is organized as follows. Section 2 provides background information on geometry computation, and presents benchmark characteristics. We review paired-single instructions in Section 3. Section 4 presents our simulation infrastructure, and Section 5 presents our simulation results. Section 6 concludes the paper.

\section{Background}

To understand the architectural aspects of geometry processing, we first describe the six stages of the $3 \mathrm{D}$ geometry pipeline. Then we characterize a set of OpenGL performance evaluation benchmarks (Viewperf [19]).

\subsection{The Geometry Pipeline}

Similar to the overall 3D computation, geometry processing can be divided into a set of pipeline stages. In a typical geometry pipeline, there are six stages as shown in Figure 1:

1. View and model transformation: graphics primitives (e.g., line, triangle or polygon) are transformed to the viewer's frame of reference. Transformations 


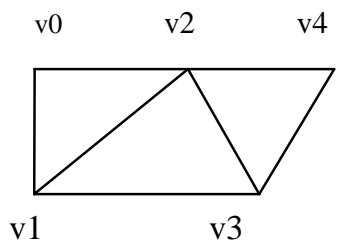

glBegin (GL_TRIANGLE_STRIP)

glVertex $3 \mathrm{fv}(\mathrm{x} 0, \mathrm{y} 0, \mathrm{z} 0)$; /* coordinates for vertex v0*/ glVertex $3 \mathrm{fv}(\mathrm{x} 1, \mathrm{y} 1, \mathrm{z} 1) ; / *$ coordinates for vertex $\mathrm{v} 1 * /$ glVertex $3 \mathrm{fv}(\mathrm{x} 2, \mathrm{y} 2, \mathrm{z} 2) ; / *$ coordinates for vertex v $2 * /$ glVertex $3 \mathrm{fv}(\mathrm{x} 3, \mathrm{y} 3, \mathrm{z} 3) ; / *$ coordinates for vertex v $3 * /$ glVertex $3 \mathrm{fv}(\mathrm{x} 4, \mathrm{y} 4, \mathrm{z} 4) ; / *$ coordinates for vertex $\mathrm{v} 4 * /$ glEnd();

Figure 2: Example of using GL_TRIANGLE_STRIP primitive.

involve vector matrix multiplication on either $1 \times 4$, $4 \times 4$ or $1 \times 3,3 \times 3$ vector and matrix sizes.

2. Lighting: the light position, color and material properties are used to calculate the object color.

3. Projection transformation: this stage determines how objects are projected to the screen. This again requires multiplication of a $1 \times 4$ vector and a $4 \times 4$ matrix.

4. Clipping: objects are clipped to the viewable area to avoid unnecessary rendering.

5. Division by w: the $\mathrm{x}, \mathrm{y}, \mathrm{z}$ components of each vertex are divided by its w component. Geometry processing usually works in the homogenous coordinate system, where all the vertices are represented with four coordinates $(\mathrm{x}, \mathrm{y}, \mathrm{z}, \mathrm{w})$.

6. Mapping vertex coordinates to screen coordinates: vertices are mapped to the screen coordinates.

Note that lighting (stage 2) is optional. For those applications that only perform wireframe rendering or implement a global illumination algorithm (i.e., the color of each vertex is precomputed), the lighting stage is unnecessary. However, the other 5 stages are mandatory.

Previous studies [1] have shown that 90 floating-point arithmetic operations are required to process a single vertex ${ }^{1}$. Current superscalar processors can issue 2 floatingpoint operations per cycle. The above analysis implies that a $500 \mathrm{MHz}$ processor could theoretically process 11 million vertices per second. This value is close to the computing capability of today's specialized hardware [17]. However, because of instruction scheduling and resource limitations, a general purpose processor is unlikely to achieve this theoretical rate. The goal of this paper is to gain further insight into these limitations. The

\footnotetext{
${ }^{1}$ Their model assumes that a single light and a viewer are at infinite distance and Gouroud shading is applied.
}

remainder of this section begins this investigation by characterizing a set of 3D applications.

\subsection{Benchmark Characterization}

To characterize the architectural aspects of 3D applications, we used ATOM's pixie tool [3] to analyze the Viewperf OpenGL performance evaluation benchmarks [19]. OpenGL is an API for graphics hardware initially defined by Silicon Graphics [24]. We use Mesa [12], a public-domain software implementation of the OpenGL specification, in this study. Mesa contains a complete software implementation of the rendering pipeline, allowing OpenGL applications to execute on machines without specialized graphics hardware.

The Viewperf suite contains five different graphic model sets including CAID (Computer Aided Industrial Design) and digital content creation models. Each set has seven to ten tests using different OpenGL primitives, lighting models and rendering parameters. In this section, we characterize three different aspects of the Viewperf benchmark set: 1) the dynamic instruction distribution, 2) the average number of vertices per glBegin/glEnd pair and 3) the amount of execution time spent in the various geometry pipeline stages.

\section{Dynamic Instruction Distribution}

The dynamic instruction distribution of the Viewperf benchmarks (average over the five different benchmarks) indicates that $42.5 \%$ of all of the instructions executed by the geometry routines involve single-precision floatingpoint instructions. A significant amount of integer instructions are needed for executing mode changes (e.g. using different texture file or changing the lighting model). The four most frequently executed instructions are load (13.4\%), multiply (12.2\%), add (9.7\%) and store (6.8\%) for single-precision floating-point data. Most of the load instructions come from loading the transform matrices and vertices. Similarly, the store instructions are used to save the processed vertices back to memory. The multiply and add instructions are primarily from the transform and lighting operations.

\section{Average Number of Vertices per glBegin/glEnd Pair}

OpenGL implements ten drawing primitives (e.g., GL_LINES, GL_TRIANGLES and GL_POLYGON). To draw an object, a set of vertices are bracketed between a call to glBegin() and glEnd(). The argument passed to glBegin() determines which geometric primitive is constructed from the vertices. 3D surfaces are usually broken down into triangles. The most efficient way for drawing a series of triangles that are connected to each other is using the GL_TRIANGLE_STRIP primitive (as shown Figure 2). However, some 3D content creation applications do 


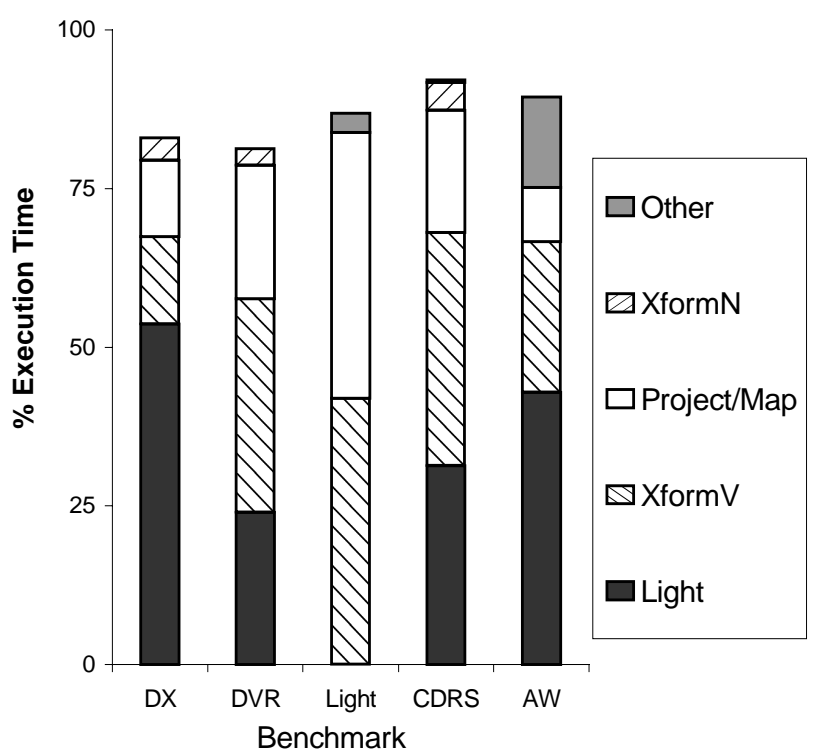

Figure 3- Execution time distribution in the MESA geometry pipeline.

not store objects in a format amenable to this drawing method. In this case, the OpenGL viewing applications may have to invoke a drawing primitive for each triangle. Thus, the number of vertices per glBegin/glEnd will be small. Profiling results show that the average number of vertices per glBegin/glEnd pair varies across the Viewperf benchmark. Awadvs uses the GL_POLYGON primitive and has only 3.4 vertices on average, while some of the CDRS tests use the GL_TRIANGLE_STRIP primitive and have up to 400 vertices per glBegin/glEnd pair.

There are four ways to exploit parallelism in geometry computation: 1) processing individual components of a vertex (e.g., coordinate (x, y, z, w) or color (R, G, B, A)), 2) processing multiple vertices of each primitive within the same pipeline stage, 3) processing vertices of each primitive in different pipeline stages and 4) processing different primitives. In the MESA implementation, the computations for vertices of each primitive (i.e., those vertices bracketed by glBegin/glEnd) in the same pipeline stage are performed in loops. Several internal library routines are executed before starting the next stage or a new set of geometry drawings.

A superscalar processor that can only exploit ILP from instructions stored in the dispatch queue is more likely to exploit the parallelism in the first two scenarios. A small number of vertices between glBegin/glEnd indicates that fewer independent floating-point instructions can be issued close in time. Thus, we do not expect benchmarks with very small number of vertices on average per glBegin/glEnd pair to achieve IPC as high as benchmarks with a large number of vertices, unless a very large dispatch queue is used.

\section{Execution Time Distribution of the Geometry Pipeline}

We divide the execution time for the geometry pipeline into five portions:

Light (gl_color_shade_vertices): ${ }^{2}$ This portion corresponds to the lighting stage, which calculates the color for each vertex.

XformV (gl_xform_normals_4fv): This portion includes the vertex transformation of both the viewing/modeling and projection transform stages. It performs multiplication of a matrix by a vector.

XformN (gl_xform_normals_3fv): This portion includes the normal vector transformation in the viewing/modeling transform stages.

Div by w/Map (gl_transform_vb_part2): This portion includes the computation of div by $\mathrm{w}$ and mapping vertex stages. It selects the appropriate lighting routine (e.g. line, polygon, and type of shading) and calls the fog, texture, and clipping routines before finally projecting the primitives to screen coordinates.

Other: This portion includes the clipping stage and the library routines executed between different pipeline stages and drawing primitives.

As shown in Figure 3, Light and XformV are the two portions where geometry processing spends the most time. Note that the Light benchmark gets its name because each vertex color is pre-computed using a global illumination algorithm, therefore, it does not actually execute the lighting functions. Awadvs spends almost $15 \%$ of the execution time in the routines executed between different pipeline stages and drawing primitives as indicated by Other in Figure 3. This is significantly higher than the other benchmarks. Awadvs has very small average number of vertices (3.4) per glBegin/glEnd pair, which implies that switching between pipeline stages and glBegin/glEnd pairs occurs more frequently.

\section{SIMD Instruction Extensions}

From the benchmark profiling discussed in the previous section, we observe that most of the arithmetic floating-point instructions are multiply and add, and these operations are all performed on single-precision values (32bit). Thus, the SIMD type instructions that perform multiply or add operations on two single-precision floating point values could fully utilize the 64-bit floating point registers in current superscalar processors and potentially eliminate a significant number of instructions. The MIPS V ISA Extension [14] proposes adding a new data type called paired-single, which packs two single precision floating-point values into one 64-bit floating-point register. The multiply and addition operations are performed

\footnotetext{
${ }^{2}$ The corresponding routine name in the Mesa implementation is listed in parenthesis.
} 


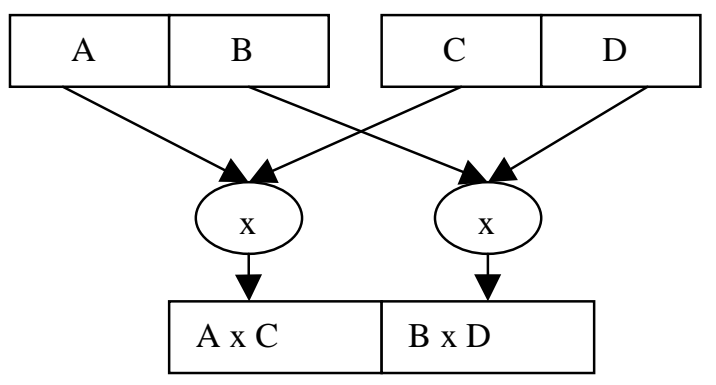

Figure 4: Operation of paired-single multiply.

on the paired-single data in the manner illustrated in Figure 4.

The SIMD instruction extensions that we consider in this paper are based on the MIPS V ISA Extensions [14]. The instruction formats and latency assumptions are summarized in Table 1. The LDPS and STPS instructions load/store a paired-single value (64 bits) from memory ignoring alignment. The PMUL (PADD/PSUB) instruction performs multiplication (addition/subtraction) of paired-single values. These paired-single instructions have 4 cycle latency and are fully pipelined. CVT.S.PL (CVT.S.PU) is used to extract the lower(higher) part of a paired-single value, and CVT.PS.S is used to create a paired-single value from two single-precision values.

The ADD_HL and LDS_HL instructions are not present in the MIPS V instruction extensions. ADD_HL adds the higher and lower parts of a paired-single value together. One example to show the usefulness of the ADD_HL instructions is the inner product operation commonly seen in the lighting stage. The inner product of two vectors $(\mathrm{x} 1, \mathrm{y} 1, \mathrm{z} 1)$ and $(\mathrm{x} 2, \mathrm{y} 2, \mathrm{z} 2)$ is $\mathrm{x} 1 * \mathrm{x} 2+\mathrm{y} 1 * \mathrm{y} 2$ $+\mathrm{z} 1^{*} \mathrm{z} 2$. The first two multiplication operations can be paired up. But the results must be added together. Without the ADD_HL instruction, we need to use the CVT.S.PU or CVT.S.PL instruction to extract the higher or lower half to a separate register before performing the addition.

The LDS_HL instruction duplicates a single-precision value to form a paired-single value. We use the computation of transforming normal vectors (multiplication of a $1 \times 3$ vector and $3 \times 3$ matrix) to illustrate the use of this instruction. The pseudo $\mathrm{C}$ codes are as follows ( $\mathrm{u}$ and $\mathrm{m}$ represent an array of vertex coordinates and the transformation matrix respectively):

$$
\begin{aligned}
& \text { for }(\mathrm{i}=0 ; \mathrm{i}<\text { number of vertices; } i++) \\
& \begin{aligned}
&\{\mathrm{q}[\mathrm{i}][0]=\mathrm{u}[\mathrm{i}][0] * \mathrm{~m}[0,0]+\mathrm{u}[\mathrm{i}][1] * \mathrm{~m}[1,0]+\mathrm{u}[\mathrm{i}][2] * \mathrm{~m}[2,0] \\
& \mathrm{q}[\mathrm{i}][1]=\mathrm{u}[\mathrm{i}][0] * \mathrm{~m}[0,1]+\mathrm{u}[\mathrm{i}][1] * \mathrm{~m}[1,1]+\mathrm{u}[\mathrm{i}][2] * \mathrm{~m}[2,1] \text {; } \\
& \mathrm{q}[\mathrm{i}][2]=\mathrm{u}[\mathrm{i}][0] * \mathrm{~m}[0,2]+\mathrm{u}[\mathrm{i}][1] * \mathrm{~m}[1,2]+\mathrm{u}[\mathrm{i}][2] * \mathrm{~m}[2,2]
\end{aligned} \\
& \}
\end{aligned}
$$

To exploit the parallelism across two vertices, we unroll the loops once and reorder instructions such that the

\begin{tabular}{|l|c|}
\hline Instruction Format & Latency(cycle) \\
\hline LDPS dest, index(base) & 2 \\
\hline PMUL src1, src2, dest & 4 \\
\hline PADD src1, src2, dest & 4 \\
\hline PSUB src1, src2, dest & 4 \\
\hline CVT.S.PL/U src, dest & 1 \\
\hline CVT.PS.S src1,src2,dest & 1 \\
\hline ADD_HL src, dest & 4 \\
\hline LDS_HL dest index(base) & 2 \\
\hline
\end{tabular}

Table 1: Instruction format and latency. All instructions are fully pipelined.

independent floating-point operations can be easily paired up. The modified version is listed below:

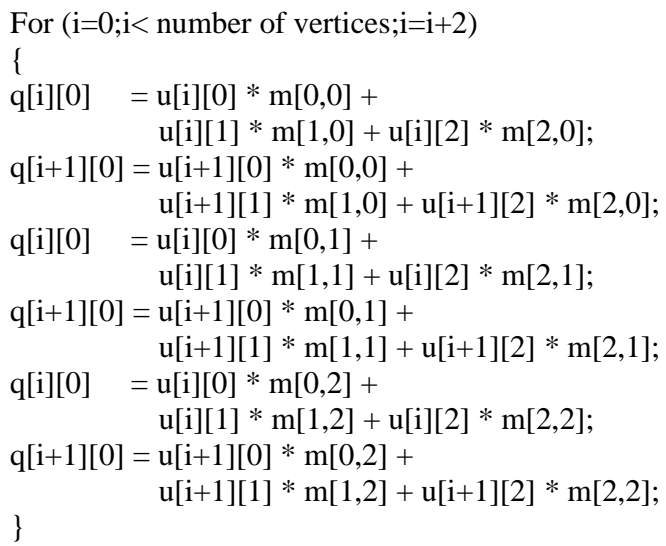

To perform the paired-single multiplication over vertices $i$ and $i+1$, we need to form paired-single values for each element of the transformation matrix (i.e., $(\mathrm{m}[0,0], \mathrm{m}[0,0]),(\mathrm{m}[1,0], \mathrm{m}[1,0]) \ldots .$.$) . The instruction$ LDS_HL is used for this purpose. Without the LDS_HL instruction, it will require one load and CVT.PS.S instruction to form each pair.

\section{Experimental Methodology}

In this section, we describe the simulation environment and processor models considered in this paper.

\subsection{Simulation Framework}

Our simulation environment (shown in Figure 5) uses ATOM [23] to perform execution-driven simulation. This simulation framework consists of two components. The first component is MESA, a software implementation of the OpenGL specification. A shared library that contains all of the routines associated with geometry computation is separated from the complete MESA implementation. ATOM allows us to instrument only this geometry library 


\begin{tabular}{|c|c|c|c|c|c|c|c|c|c|c|}
\hline \multirow{2}{*}{$\begin{array}{l}\text { Processor } \\
\text { Model }\end{array}$} & \multirow{2}{*}{$\begin{array}{c}\text { Total } \\
\text { Issue Width }\end{array}$} & \multicolumn{4}{|c|}{ \# of Integer Functional Units } & \multicolumn{5}{|c|}{ \# of Floating-Point Functional Units } \\
\hline & & $\begin{array}{l}\text { Issue } \\
\text { Limit }\end{array}$ & $\begin{array}{l}\text { loads\& } \\
\text { stores }\end{array}$ & $\begin{array}{l}\text { Control } \\
\text { flow }\end{array}$ & other & $\begin{array}{l}\text { Issue } \\
\text { Limit }\end{array}$ & mul & div & sqrt & other \\
\hline Base & 4 & 4 & 2 & 2 & 4 & 2 & 1 & 1 & 1 & 1 \\
\hline $2 \times B$ ase & 8 & 8 & 4 & 4 & 8 & 4 & 2 & 1 & 1 & 2 \\
\hline 4xBase & 16 & 16 & 8 & 8 & 16 & 8 & 4 & 1 & 1 & 4 \\
\hline $2 \mathrm{xFP}$ & 6 & 4 & 2 & 2 & 4 & 4 & 2 & 1 & 1 & 2 \\
\hline $4 \mathrm{xFP}$ & 10 & 4 & 2 & 2 & 4 & 8 & 4 & 1 & 1 & 4 \\
\hline
\end{tabular}

Table 2: Instruction issue rules (ready instructions are issued in fetch order).

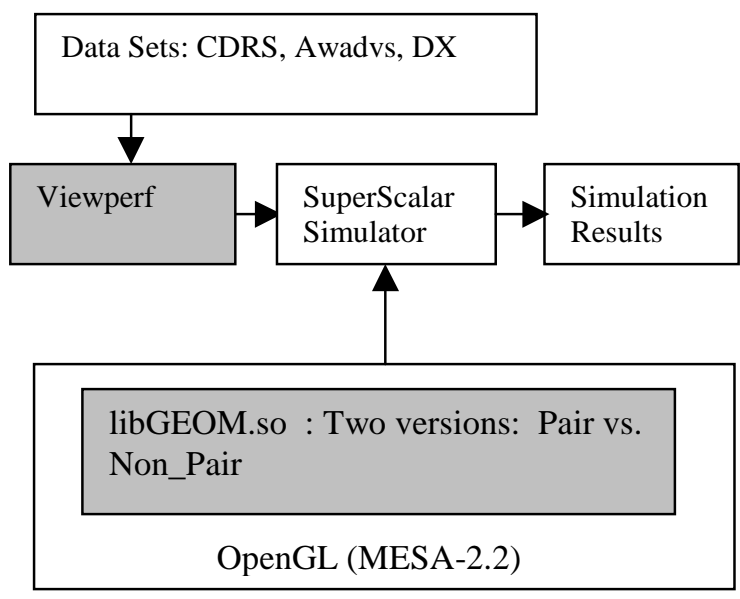

Figure 5: Simulation framework. libGEOM.so is a shared library including all the routines associated with the geometry processing. We only instrument code in the highlighted boxes.

and the application itself. In this way, we can simulate the environment where the host CPU is responsible for database traversal and geometry processing, while specialized hardware is used to process the remaining tasks in the graphics pipeline. We modified four routines, which account for $75 \%$ to $90 \%$ of the total execution time for all the benchmarks we ran, to incorporate paired-single instructions. These routines correspond to the Light, XformV, XformN and Div by w/Map as described in Section 2.2.

The second component of our simulation framework is an ATOM-based simulator that models an out-of-order superscalar processor with speculative execution [4], whose instruction set is based on the DEC Alpha processor [22].

To simulate the new instructions, we place innocuous (but unique) "marker" instructions where we want to replace the original code with new instructions. The operands of the marker instructions indicate different instruction types (e.g., LDPS, MULPS, etc). The appropriate operands of the new instructions are passed through the next 2 or 3 marker instructions, depending on the number

\begin{tabular}{|l|l|c|c|}
\hline \multicolumn{2}{|l|}{ Instruction Type } & latency & pipeline \\
\hline Integer & multiplication & 6 & yes \\
\cline { 2 - 4 } & load & 2 & yes \\
\cline { 2 - 4 } & store & 1 & yes \\
\cline { 2 - 4 } & control flow & 1 & yes \\
\cline { 2 - 4 } & other & 1 & yes \\
\hline \multirow{4}{*}{$\begin{array}{l}\text { Floating- } \\
\text { point }\end{array}$} & $32-$-bit div & 8 & no \\
\cline { 2 - 4 } & 64-bit div & 16 & no \\
\cline { 2 - 4 } & square root & 33 & no \\
\cline { 2 - 4 } & other & 4 & yes \\
\hline
\end{tabular}

Table 3: Instruction latencies.

of the operands required. In this way, instruction dependencies are accurately maintained. The simulator decodes each instruction and takes appropriate actions to simulate the paired-single execution when it encounters the marker instruction.

\subsection{Processor Models}

The baseline processor model studied in this paper is a 4-way, out-of-order issue superscalar processor. The issue rules and the functional unit latencies are summarized in Table 2 and Table 3. The maximum number of instructions that can be inserted into the dispatch queue or committed is equal to the issue width. We assume a perfect memory system (i.e., every memory reference and instruction fetch hit in the L1 cache) $)^{3}$, a unified dispatch queue and separate register files for the integer and floating-point functional units. Speculative execution is enabled by implementing the branch prediction scheme proposed by McFarling [11] and precise exceptions are imposed.

To investigate the effect of a wider issue superscalar processor on the performance of geometry processing, we examine the following 4 models: $2 x B a s e, 4 x B a s e, 2 x F P$ and $4 \mathrm{xFP}$ as listed in Table 2 . The $2 \times \mathrm{xBase}$ and $4 \mathrm{xBase}$ models are 8-way and 16-way issue processors respec-

\footnotetext{
3 The miss rates for a 64-K, 2-way set associative D-cache and 8-K direct-mapped I-cache are both less than $2 \%$ for most of the benchmarks. Thus, we assume a perfect memory system to reduce simulation time.
} 

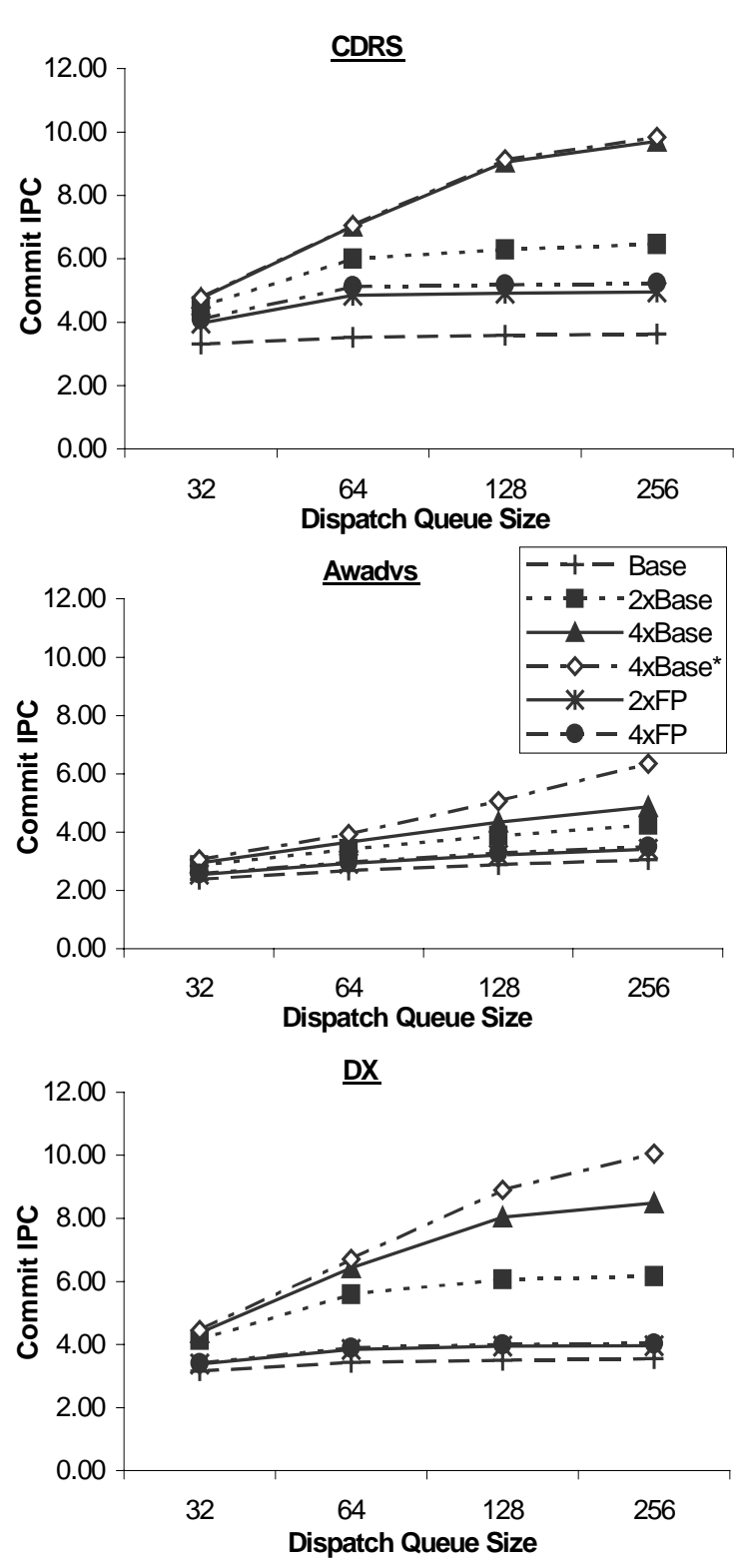

\section{Figure 6: The commit IPC of various processor models with varying dispatch queue size.}

(4xBase* represents the issue IPC of the $4 x$ Base processor)

tively. The issue rules are similar to the Base model. However, for most instruction types, two or four times the number can be issued in one cycle. The exceptions are division and square root, which remain the same as the baseline model. The reason for not doubling these two functional units is for a fair performance comparison between 2xFP and a baseline processor with the pairedsingle instruction since the paired-single operations are not implemented for division and square root. For the $2 \mathrm{xFP}(4 \mathrm{xFP})$ configurations, we double (quadruple) only the floating-point functional units and issue width. The number of the integer functional units remains the same as the baseline processor. Then total issue width becomes 6 and 10 for $2 \mathrm{xFP}$ and $4 \mathrm{xFP}$ respectively.

\section{Simulation Results}

We use CDRS, Awadvs and DX from Viewperf as our benchmarks due to lengthy simulation time. Each of these benchmarks is composed of several tests. For space reasons, we only present test 1 from each benchmark. These three tests are chosen because they are representative of all the tests (the complete simulation results are provided in [25]). CDRS test 1 is a wireframe rendering application and both DX and Awadvs have at least one light source. Awadvs has only 3.4 vertices on average per glBegin/glEnd, while CDRS and DX test1 have 30 and 96 vertices respectively.

We present our simulation results in three parts. First, we investigate how well conventional superscalar processors exploit the parallelism in geometry processing. Then we present the performance of paired-single execution on different processor models. Finally, we compare the relative performance of different processors with and without paired-single instructions accounting for potential increases in CPU clock cycle time.

\subsection{Scaling Conventional Design}

The dispatch queue and register file sizes have significant impact on how much ILP can be exploited in a superscalar processor. A wider issue machine usually requires a larger dispatch queue and register file. In order to evaluate the potential performance improvement achieved by increasing the issue width, the superscalar simulator is first configured with 2048 floating-point and 2048 integer registers. With such a large register file, the CPU never stalls due to a lack of free registers. We then vary the dispatch queue size from 64 to 256 . The commit IPC ${ }^{4}$ for the various processor models is shown in Figure 6.

CDRS test1 has the highest IPC for all the configurations among all the benchmarks we ran. With the largest dispatch queue (256), the commit IPC of 2xBase (8-way issue) is 6.5, almost twice that of Base (3.4). Doubling only the floating-point issue width (2xFP) achieves $36 \%$ performance improvement. However, quadrupling only the floating-point issue width $(4 \mathrm{xFP})$ does not perform any better than the $2 x F P$ because the loads that read the source operands for the floating-point operations become the bottleneck. The commit IPC of the $4 \mathrm{xBase}$ processor (16-way issue) is 9.7, about 2.7 times that of Base.

The continuous growth of commit IPC as the issue width increases indicates that a lot of parallelism does exist in geometry processing for this benchmark. Note

\footnotetext{
${ }^{4}$ The commit IPC is the ratio of the number of instructions that commit to the total execution cycles.
} 

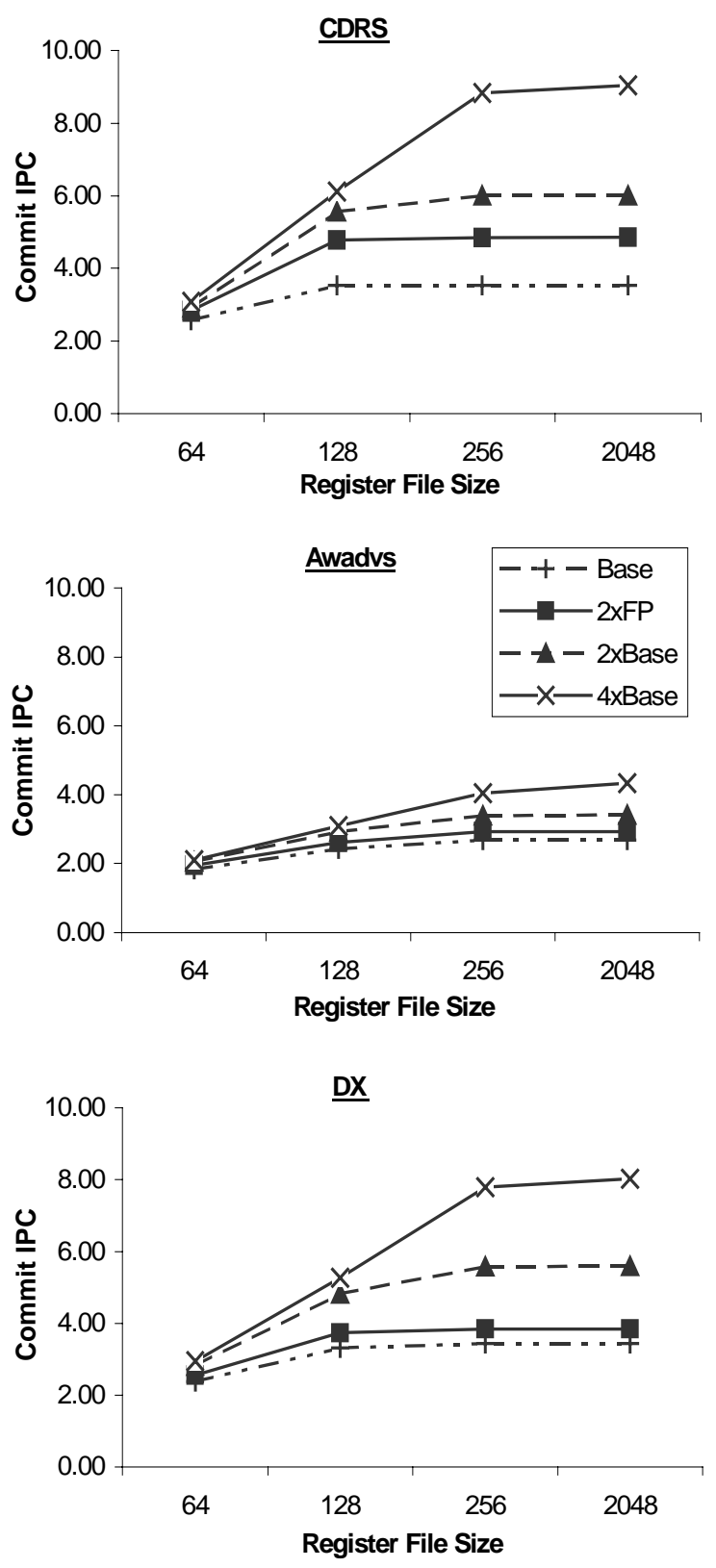

Figure 7: The commit IPC for various processor models with varying register file size.

that the commit IPC grows with larger dispatch queue size, but the degree of improvement diminishes after a certain size. This point occurs around a dispatch queue size of 32 for the Base model, 64 for both the 2xBase and $2 x F P$, and 128 for $4 x B a s e$.

For DX test1, the commit IPC of the processor models smaller than $4 x B a s e$ are comparable to CDRS test1, except for $2 \mathrm{xFP}$, which only achieves $13 \%$ performance improvement. The ratio of floating-point arithmetic operations to load instructions is 1:1 for DX test1 and 2:1 for
CDRS test1. Thus, increasing the floating-point issue width alone does not improve the performance of DX as much as that of CDRS. For DX test1, the commit IPC of $4 \times$ Base is 8.48, lower than CDRS test1 (9.7). The lower commit IPC is due to more mispredicted branches. The issue IPC for $4 \mathrm{xBase}$ is plotted in Figure 6 to illustrate this scenario. Issued instructions can not commit if a preceding branch in the program order was mispredicted. DX test1 has a larger difference between the issue and commit IPC than CDRS test1. This is because the lighting computation has more conditional branches than the transform, thus the performance of a light-intensive applications like DX test1, is more subject to branch prediction accuracy than a wireframe rendering application like CDRS test1.

Awadvs test 1 has the lowest IPC, primarily because of its small number of vertices (3.4) per glBegin/glEnd. Observe that the commit IPC increases linearly with the dispatch queue size, hence, for this benchmark, the dispatch queue is still the bottleneck even when it has 256 entries. Because the $4 \mathrm{xFP}$ processor performs equal to $2 x F P$ for all the benchmarks we ran, we no longer consider this configuration in the following analysis.

To evaluate how the register file size affects performance, we keep the dispatch queue size constant (64 entries for Base, $2 \mathrm{xFP}$ and $2 \mathrm{xBase}$ and 128 entries for $4 \mathrm{xBase}$ ) while varying the register file size from 64 to 256 . The results are shown in Figure 7. The 2048 entry register file size is shown as a reference point. Using more than 128 registers for Base, $2 \mathrm{xFP}$ and $2 \mathrm{xBase}$ and 256 registers for $4 x$ Base does not improve performance significantly.

In the next section, we analyze the benefit of the paired-single execution on the Base, $2 \mathrm{xFP}$ and $2 \mathrm{xBase}$ processors. $4 \times$ Base is a 16-way issue machine and requires a 128 -entry dispatch queue and 256 registers. This configuration is too large to achieve a practical implementation by simply scaling the Base configuration, hence we do not consider it further.

\subsection{The Performance Improvement of Paired- Single Execution}

Adding paired-single instructions not only reduces the number of single-precision floating-point add and multiply instructions, it can also eliminate load/store instructions if the LDPS instruction can be used to load two single-precision floating-point values together. Table 4 shows the reduction amount for each instruction type. The number of multiply and add instructions is reduced by approximately $50 \%$ for CDRS test $1,40 \%$ for DX and Awadvs test 1 . The number of load and store instructions are reduced by $5 \%$ to $15 \%$ and $15 \%$ to $36 \%$, respectively. CDRS has the highest overall instruction reduction of $17 \%$.

Reducing the number of instructions has two potential advantages. First, combining two floating-point opera- 


\begin{tabular}{|l|l|l|l|l|l|l|l|l|l|l|l|l|l|l|l|}
\hline Benchmark & \multicolumn{4}{|c|}{ CDRS } & \multicolumn{4}{|c|}{ DX } & \multicolumn{4}{|c|}{ Awadvs } \\
\hline $\begin{array}{l}\text { Instruction } \\
\text { Type }\end{array}$ & add & mul & ld & sts & total & add & mul & ld & sts & total & add & mul & lds & sts & total \\
\hline Reduction $\%$ & 48 & 47 & 5 & 36 & 17 & 43 & 39 & 9 & 15 & 7 & 40 & 37 & 15 & 21 & 11 \\
\hline
\end{tabular}

Table 4: Instruction reduction of different instruction type for paired-single execution.

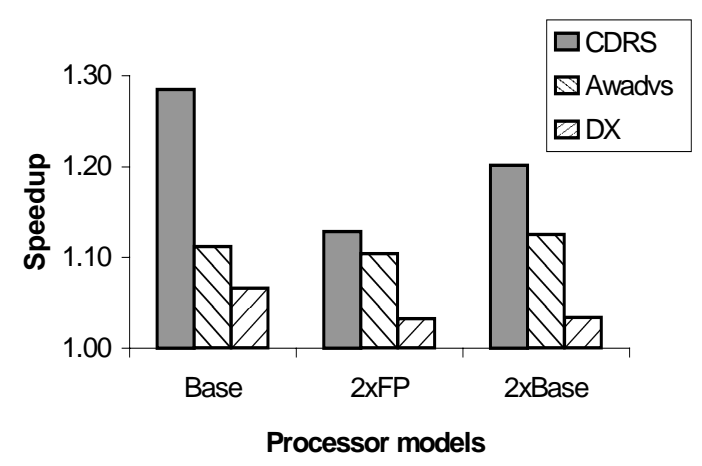

Figure 8: Performance improvement of pairedexecution.

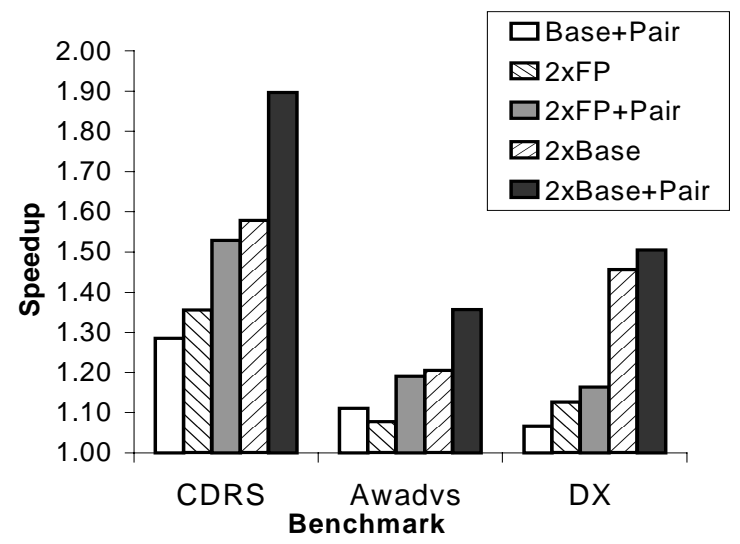

Figure 9: Relative speedup of various processor models over the Base with a dispatch queue of 64 entries and 128 registers.

tions together effectively enables the CPU to look further ahead to find independent instructions to issue. In other words, adding paired-single instructions could achieve the same effect as increasing the dispatch queue size. Second, it can improve the instruction cache performance. We did not analyze this due to the low instruction cache miss rate for the benchmarks we ran. We can expect higher performance impact on an embedded system, which is usually configured with a smaller instruction cache.

We evaluate the performance improvement of pairedsingle execution on the Base, 2xFP and 2xBase models. The simulation results are shown in Figure 8. The y-axis shows the speedup of paired-single over non-paired execution. CDRS test 1 has the best performance improvement, $28 \%$ on the Base model, $13 \%$ on the $2 \mathrm{FP}$ and $20 \%$ on the $2 \mathrm{xBase}$. DX test1 has the smallest performance improvement since it only reduces the number of instructions by $7 \%$. Note that our speedups may not be optimal. First, there are some routines required for geometry processing that we have not converted to use paired-single instructions. However the impact on performance of these procedures should not be substantial. Second, we have not optimized the instruction schedule of the pairedsingle sequence. Different computation sequences incur different register allocation and instruction scheduling, and analyzing these effects requires further research.

\subsection{Paired-Single vs. Wider Issue}

In this section, we discuss the relative performance of various processor models with and without the pairedsingle instruction set. First, we compare relative performance assuming that CPU cycle time remains the same for all processor models. Then, we investigate how changes in cycle time affect overall performance. All the processor models are configured with a 64-entry dispatch queue and 128 registers. These numbers are chosen such that the performance of an 8-way issue (2xBase) processor is not constrained too much by the dispatch queue and register file and the processor configuration is within a reasonable range.

The simulation results, assuming no changes in CPU cycle time, are shown in Figure 9. The y-axis is the speedup of the various processor models over the Base configuration. Adding paired-single instructions effectively doubles the floating-point issue width so a Base processor with the paired-single instruction extension can potentially achieve the same floating-point processing capability as $2 \mathrm{xFP}$.

Our results show that Base+Pair performs within $7 \%$ of 2xFP for CDRS and DX and it even outperforms 2xFP for Awadvs test1. Besides the advantage of doubling floating-point processing rate, adding paired-single instructions can better utilize the dispatch queue, as mentioned in the previous section. For an application where the dispatch queue is the performance bottleneck, like Awadvs test1, Base+Pair has a performance advantage over 2 xFP. An 8 -way issue processor using paired-single instructions (2xBase+Pair) can achieve 1.9 speedup over Base for CDRS test1. 


\begin{tabular}{|l|c|c|}
\hline $\begin{array}{l}\text { Processor } \\
\text { Model }\end{array}$ & $\begin{array}{l}\text { Register File } \\
\text { Access Time(\%) }\end{array}$ & $\begin{array}{l}\text { Issue Logic } \\
\text { Delay (\%) }\end{array}$ \\
\hline 2 xFP & 0 & $7 \%$ \\
\hline 2xBase & $50 \%$ & $14 \%$ \\
\hline
\end{tabular}

Table 5: Cycle time increase over the Base model assuming 0.35um technology.

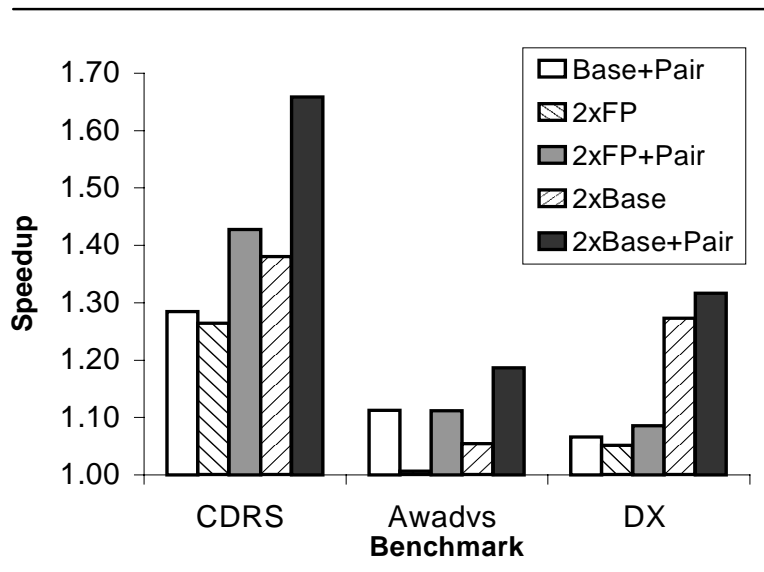

Figure 10: Relative performance of various processor models assuming that the window issue logic determines processor cycle time.

\subsubsection{Effects on Clock Cycle Time}

Previous studies have shown that increasing issue width has significant impact on the processor cycle time [4][20]. Palacharla et al. [20] studies how the instruction dispatch, issue logic and data bypass delay varies with different issue width. Their results show that the issue logic determines the critical path delay in a 0.35 um technology for both 4-way and 8-way issue processors (not considering cache and register files) and the wakeup logic delay (part of issue logic) grows linearly with the issue width. Farkas et al. [4] shows that the issue width determines the number of read/write ports to a register file, and thus can have significant impact on the cycle time.

The percentage increase of issue logic delay and register file access time over the Base model are summarized in Table 5. We use a modified version of CACTI [7] developed by K. Farkas in [4] to generate the register file access time. Note that the floating-point register file of $2 \mathrm{xFP}$ has the same number of read/write ports as the integer register file of Base. Thus, the register file access time of $2 \mathrm{xFP}$ is equal to the Base access time. The $2 x$ Base model increases the register file cycle time by $50 \%$ in a $0.35 \mathrm{mu}$ technology. We use the data provided in [21] to derive the issue logic delay. Linear extrapolation is used to obtain the data for configurations not studied in

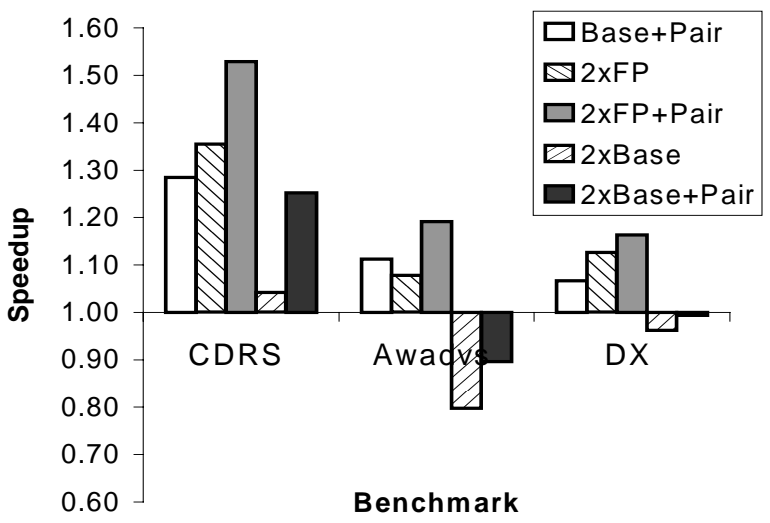

Figure 11: Relative performance of various processor models assuming that the register file access delay determines processor cycle time.

that paper. The 2xFP model increases the issue logic delay by $7 \%$, and 2 xBase by $14 \%$.

We present simulation results in two sets. The first set assumes that issue logic (wakeup+selection) determines the critical path delay (Figure 10) and the second set assumes that register file access does (Figure 11). The yaxis is the speedup of the various processor models over the Base configuration. If the issue logic determines critical path delay, Base+Pair outperforms 2xFP for all the benchmarks. The performance difference is most substantial for Awadvs test1. The 2xBase+Pair processor model achieves 1.6 speedup for CDRS test1. However, if the CPU cycle time is determined by the register file access, increasing the issue width up to 8 -way (2xBase) has negative impact on the performance for Awadvs and DX test1 as shown in Figure 11. Thus, 2FP+Pair becomes the best design choice, achieving 1.5 speedup over the Base processor model for CDRS and 1.2 for both Awadvs and DX.

\section{Conclusion}

The widespread use of multi-media applications presents new design challenges for system designers. In this paper, we examine the performance of geometry computation in three dimensional graphics applications on future superscalar processors. Geometry computation is singleprecision (32-bit) floating-point intensive. We investigate the performance of recently proposed instructions that perform two independent 32-bit operations by packing the operands in 64-bit registers and exploiting the existing 64bit datapath. We use simulation to compare the performance of these new instructions, called paired-single to that achieved by increasing a conventional out-of-order processor's issue-width. 
From our simulation results, we found that pairedsingle instructions improve performance by up to $28 \%$ on a 4 -issue processor and $20 \%$ on an 8 -issue. These improvements are comparable to those achieved by doubling only the floating-point issue width (2xFP). Our results reveal that $4 \mathrm{xFP}$ performs equal to $2 \mathrm{xFP}$ because load instructions that read source operands of floating-point operations become the bottleneck, and hence require a commensurate increase in the integer issue width.

We also found that the average number of vertices processed in each stage of the geometry pipeline (i.e., vertices per glBegin/glEnd) is the primary factor determining performance on superscalar processors. For benchmarks that have a large number of vertices per glBegin/glEnd, the speedup of an 8-way issue processor over a 4-way is 1.6 with a 64-entry dispatch queue and 128 registers. However, for benchmarks that have a small average number of vertices per glBegin/glEnd, the speedup is only 1.2.

Considering the impact of the issue width on the CPU cycle time, we looked at two pipeline stages that can be on the critical timing path. One is the register file access and the other is the issue logic. If the issue logic is on the critical path, an 8-way issue processor with paired-single instructions provides $20 \%$ to $65 \%$ performance improvement over a 4-way issue. However, if the register file access is on the critical path, the processor cycle time increases by almost $50 \%$ going from 4 -way to 8 -way. Thus doubling only the floating-point issue width of a 4-issue processor (2xFP) with paired-single instructions becomes the best design choice. The improvement over a 4-way issue processor ranges from $20 \%$ to $50 \%$.

\section{Acknowledgements}

We thank Keith Farkas for providing his superscalar simulator and his comments and suggestions on this work. Special thanks go to Subbarao Palacharla for his helpful answers to our questions about the clock cycle time analysis. We also thank Norman Jouppi, Gershon Kedem, David Raymond, Srikanth Srinivasan, Mithuna Thottethodi, and the anonymous reviewers for providing useful comments on this paper.

\section{References}

[1] K. Akeley, and T. Jermoluk. High-Performance Polygon Rendering. Computer Graphics, Volume 22, pages 239249, August 1988.

[2] AMD 3DNow! Technology. http://www.amd.com/product/cpg/k623d/inside3d.html

[3] Digital Unix V4.0 Programmer's Guide, pages 8-13.

[4] K. Farkas. Memory-system Design Considerations for Dynamically-scheduled Microprocessors. Ph.D. thesis, Department of Electrical and Computer Engineering, University of Toronto, January 1997.
[5] J. Foley, A. van Dam, S. Feiner, and J. Hughes. Computer Graphics - Principles and Practice. Addison-Wesley, 1996.

[6] Intel MMX2. http://developer.intel.com/drg/news/katmai.html.

[7] N. Jouppi and S. Wilson. An enhanced access and cycle time model for on-chip caches. Technical Report 93.5, DEC Western Research Laboratory, July 1994.

[8] G. Kane. PA-RISC 2.0 Architecture. Prentice Hall PTR, 1996.

[9] L. Kohn, G. Maturana, M, Tremblay, A. Prabhu, and G. Zyner. Visual Instruction Set (VIS) in UltraSPARCTM. In Proceedings of COMPCON'95, pages 462-469, March 1995.

[10] R. Lee and M. Smith. Media Processing: A New Design Target. IEEE Micro, pages 6-9, August 1996.

[11] S. McFarling. Combing Branch Predictors. Digital Equipment Corporation Western Research Lab Technical Note TN-36, 1993

[12] MESA library. http://www.ssec.wisc.edu/ brianp/Mesa.html

[13] Microprocessor Forum, October 1997.

[14] MIPS V ISA Extension. http://www.sgi.com/MIPS/arch/ISA5/

[15] MMX ${ }^{\mathrm{TM}}$ Technology. Intel Architecture MMX Technology Programmer's Reference Manual. Intel Corporation, March 1996

[16] S. Molnar, J. Eyles, and J. Poulton. PixelFlow: High-Speed Rendering Using Image Composition. In Proceedings of SIGGRAPH '92, pages 231-240, August 1992.

[17] J. Montrym, D. Baum, D. Dignam, and C. Migdal. InfiniteReality: A Real-Time Graphics System. In Proceedings of SIGGRAPH '97, pages 293-302, August 1997.

[18] Motorola AltiVec Technology. http://www.mot.com/SPS/PowerPC/AltiVec

[19] OpenGL Performance Benchmark - Viewperf. http://www.specbench.org/gpc/opc.static/vp50.html

[20] S. Palacharla, N. Jouppi, and J. Smith. Complexity -Effective SuperScalar Processors. In Proceedings of the $24^{\text {th }}$ Annual International Symposium on Computer Architecture, pages 206-218, 1997.

[21] S. Palacharla, N. Jouppi, and J. Smith. Quantifying the Complexity of Superscalar Processors. Technical Report CS-TR-96-1328, University of Wisconsin-Madison, November 1996.

[22] R. Sites. Alpha Architecture Reference Manual. Digital Press, 1992.

[23] A. Srivastava and A. Eustace. ATOM: A system for building customized program analysis tools. In Proceedings of the ACM SIGPLAN '94 Conference on Programming Languages, March 1994.

[24] M. Woo, J. Neider, and T. Davis. OpenGL Programming Guide. Addison-Wesley, 1997.

[25] C. Yang, B. Sano, and A. Lebeck. Exploiting Instruction Level Parallelism in Geometry Processing for Three Dimensional Graphics Applications. Technical Report CS1998-14, Computer Science Department, Duke University, September 1998. 